\title{
Trailing Edge Noise Reduction by Passive and Active Flow Controls
}

\author{
Jung Hoon Kim ${ }^{1}$, Al-Sadawi Laith ${ }^{2}$, Alexandros Vathylakis ${ }^{3}$ and Tze Pei Chong ${ }^{4}$ \\ School of Engineering and Design, Brunel University, Uxbridge, UB8 3PH, UK
}

This paper presents the results on the use of porous metal foams (passive control) and dielectric barrier surface plasma actuations (active control) for the reduction of vortex shedding tonal noises from the nonflat plate type trailing edge serration in a NACA0012 airfoil previously discussed in Chong et al. (AIAA J. Vol. 51, 2013, pp. 2665-2677). The use of porous metal foams to fill the interstices between adjacent members of the sawtooth can almost completely suppress the vortex shedding tonal noise, whilst the serration effect on the broadband noise reduction is retained. This concept will promote the nonflat plate type serrated trailing edge to become a genuine alternative to the conventional flat plate type serrated trailing edge, which is known to have drawbacks in the structural stability, aerodynamic performances and implementation issues. For the plasma actuators, configuration which produces electric wind in a tangential direction is found to be not very effective in suppressing the vortices emanated from the serration blunt root. On the other hand, for the plasma configuration which produces electric wind in a vertical direction, good level of vortex shedding tonal noise reduction has been demonstrated. However, the self noise produced by the plasma actuators negates the noise benefits on the tonal noise reduction. This characteristic illustrates the need to further develop the plasma actuators in a two pronged approach. First is to increase the electric wind speed, thereby allowing the plasma actuators to be used in a higher free jet velocity which naturally produces a larger level of jet noise. Second, the self noise radiated by the plasma actuators should be reduced.

\section{Nomenclature}

$\mathrm{b}=\quad=$ thickness of flat plate, $\mathrm{mm}$

$C \quad=$ chord length, $\mathrm{mm}$

$f=$ frequency, $\mathrm{Hz}$

$2 \mathrm{~h}=$ serration amplitude (root-to-tip distance), $\mathrm{mm}$

SPL $=$ Sound Pressure Level, $\mathrm{dB}$

$x=$ a coordinate with streamwise direction from the trailing edge, $\mathrm{mm}$

$\lambda=$ serration period, $\mathrm{mm}$

$\varphi \quad=$ serration angle, degree

\footnotetext{
${ }^{1}$ Post-doctoral research fellow, School of Engineering and Design, Junghoon.kim@brunel.ac.uk, AIAA Member

${ }^{2} \mathrm{PhD}$ student, School of Engineering and Design, laith.al-sadawi@ brunel.ac.uk, Non-AIAA Member

${ }^{3} \mathrm{PhD}$ student, School of Engineering and Design, alexandros.vathylakis@ brunel.ac.uk, AIAA Student Member

${ }^{4}$ Lecturer, School of Engineering and Design, t.p.chong@ brunel.ac.uk, AIAA Member
} 


\section{Introduction}

$\mathrm{I}^{\mathrm{n}}$ $\mathrm{n}$ the middle of the last century, jet engine noise has been considered as the major source of aircraft noise. Since then, the technology has progressed so much that significant reduction in jet noise can be achieved by the high bypass ratio turbofan engines. More attention is now paid to other noise sources such as those generated at the trailing edge of fan blade or airframe's high lift devices. The dominant noise mechanism is by the scattering of the hydrodynamic pressure fluctuations near the trailing edge. In the context of a high Reynolds number flow at low pressure loading configuration, turbulent boundary layer tends to develop on the aerofoil surface. The radiated noise from the trailing edge will therefore be largely broadband in nature.

There has been much interest recently in developing control methods aimed at reducing the aforementioned trailing edge broadband noise. Approaches taken include serrated edges ${ }^{1-3}$, porous surface ${ }^{4}$ and brushes ${ }^{5-6}$. These passive methods have been demonstrated experimentally in low speed rig tests to afford good level of radiated noise reduction. One of the most promising treatments has been the use of serrated trailing edges. In nearly all cases, however, the serrations have been cut into flat plates and then inserted into the trailing edge of the main aerofoil body. Questions remain whether the flat plate type serration has the acceptable structural strength and integrity to continuously operate in a high pressure loading configuration, as well as whether it will alter the aerodynamic performance of the original aerofoil.

Another type of the trailing edge device is to cut the serrations directly into the aerofoil body, which has also been shown to afford good level of broadband noise reduction ${ }^{7}$. However, the overall performance of the nonflat plate type serrated trailing edge is overshadowed by the bluntness-induced vortex shedding tonal noise, and the drag is also possibly increased. Despite the previous attempt to minimise the bluntness-induced vortex shedding ${ }^{8}$, the nonflat plate type serration is still not a genuine alternative to the flat plate type serration.

In this paper, the bluntness-induced vortex shedding noise of a nonflat plate type serrated trailing edge will be suppressed by a two pronged approach. The first approach utilises porous metal foams to fill the interstices between adjacent members of the sawtooth. The convoluted and corrugated fluid pathways within the metal foam could be used to inhibit the vortex shedding provided that the solidity ratio is adequate. On the other hand, if the solidity ratio of the metal foam is too high it will tend to fill the interstices so completely that the serrated pattern of the trailing edge will be lost. In this work, a metal foam of about 5\% solidity ratio returns a very promising result whereby the vortex shedding tonal noise can be suppressed almost completely.

The second approach for the flow and noise control is to produce periodic perturbations at each interstice between adjacent members of the sawtooth by the Dielectric Barrier Discharge (DBD) plasma actuators. These actuators, in their simplest form, consist of two electrodes and a dielectric material separating the two electrodes. When a sufficient AC electric current is applied at the exposed electrode an electric field between the two electrodes will be formed, which will cause ionization of the air particles above it and an electric wind will be produced in the spanwise or streamwise direction, depending on the orientation of the electrodes. In the current work, a DBD plasma actuator will be utilised to transfer momentum through collision between charged and neutral air particles into the boundary layer in the vicinity of the trailing edge. As a result of such transmission of momentum, a reduction of the vortex shedding tonal noise may be possible. The plasma actuators were first tested on a flat plate with a twodimensional blunt trailing edge in order to determine the most effective plasma actuator configuration that could then be applied to the more complex nonflat plate serrated trailing edge of the aerofoil.

Note that the results presented in this paper are mainly for the demonstration of the passive and active flow controls on the reduction of the bluntness-induced vortex shedding tonal noise. Detailed investigation of the noise reduction mechanism has not been attempted yet and is not the objective of this paper.

\section{Experimental setup}

\section{A. Aeroacoustic wind tunnel facilities and instrumentations}

Free field measurements of the aerofoil noise with the passive flow control treatment (porous metal foams on each interstice) were conducted in the DARP rig in the Institute of Sound and Vibration Research (ISVR) at University of Southampton. The DARP rig is situated in a large anechoic chamber of $8 \mathrm{~m} \times 8 \mathrm{~m} \times 8 \mathrm{~m}$. The nozzle exit is a rectangular section with a dimension of $0.15 \mathrm{~m}$ (height) $\times 0.45 \mathrm{~m}$ (width). The turbulence intensity of this wind tunnel is about $0.1 \%$ and the background noise level is very low. More details about this facility can be found

in ${ }^{9}$. The range of jet speed under investigation was between $20-60 \mathrm{~ms}^{-1}$. The aerofoil is held by side plate and set at zero degree angle of attack. Far field noise measurement was made by a $1 / 2$ inch $B \& K$ condenser microphone at a distance of $1.5 \mathrm{~m}$ directly above the aerofoil's trailing edge at mid span. Noise data was sampled at $44 \mathrm{kHz}$ for 20 
seconds by a 24-bit analogue-digital card (National Instruments). The acquired data were then windowed-FFT and averaged to obtain the power spectral density with a resolution of $1 \mathrm{~Hz}$.

The plasma actuator experiment was conducted in the newly commissioned aeroacoustic wind tunnel at Brunel University ${ }^{10}$. The open nozzle is situated inside a hemi-anechoic chamber with a dimension of $4 \mathrm{~m}$ x $5 \mathrm{~m} \times 3.4 \mathrm{~m}$. The air flow is generated from the centrifugal fan outside the anechoic chamber and the flow is acoustically treated by a $10 \mathrm{~m}$ long silencer located above the ceiling of the chamber. After the air leaves the silencer, it enters the settling chamber and then accelerates through the nozzle, which is situated inside the anechoic chamber. The nozzle exit is a rectangular section with a dimension of $10 \mathrm{~cm}$ (height) $\mathrm{x} 30 \mathrm{~cm}$ (width). The exhausted jet velocity is uniform with discrepancy of less than $1 \%$, and the turbulence intensity is between $0.1-0.2 \%$. The aeroacoustic wind tunnel produces very low background noise ${ }^{11}$. Both the flat plate and aerofoil models can be interchanged with the side walls extending from the nozzle. A $1 / 2$ inch free field type of pre-polarised condenser microphone (LarsonDavis 377B02) and a preamplifier (LarsonDavis 426E01) is used to measure the noise. The microphone is installed at a distance of $1 \mathrm{~m}$ above the aerofoil trailing edge at mid span. The noise data was sampled at $44 \mathrm{kHz}$ by a 16-bit analogue-digital card from National Instrument. The acquired data were then windowed-FFT and averaged to obtain the power spectral density with a resolution of $1 \mathrm{~Hz}$.
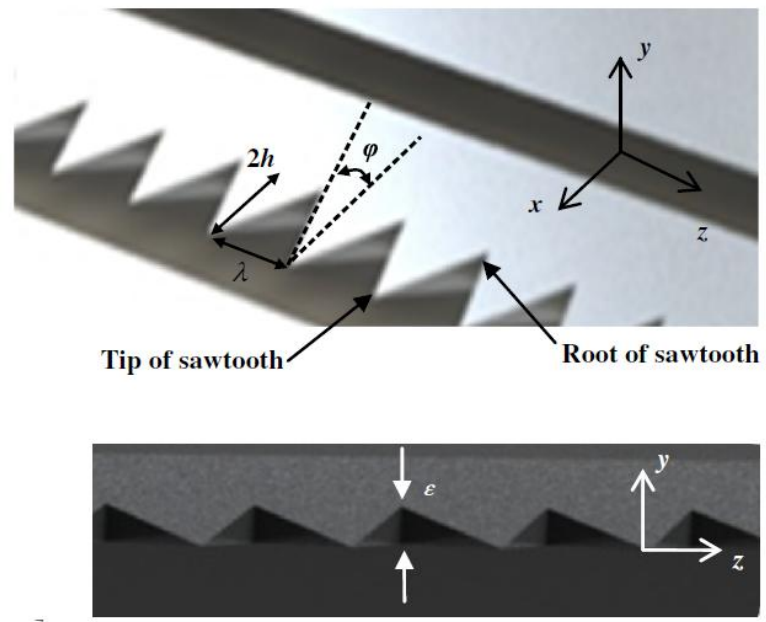

Figure 1. Illustrations of the sawtooth geometry adopted in the current study for the nonflat plate type trailing edge serration

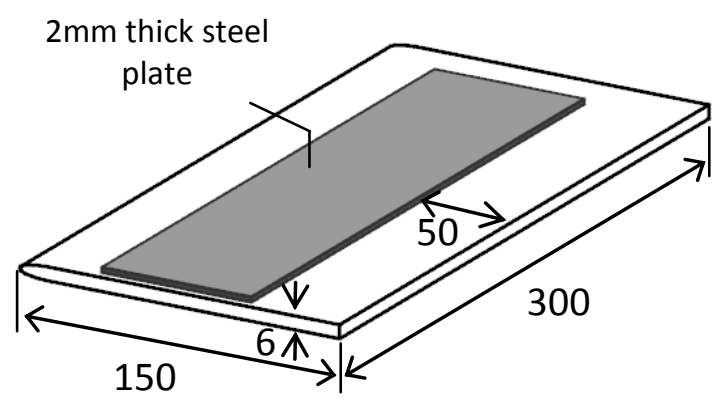

Figure 2. Schematic of the flat plate model used for the FP-PA1 and FP-PA2 plasma actuators

\section{B. Test models}

Similar to the previous works $\mathrm{in}^{7,8}$, the test model is a NACA0012 aerofoil with a nonflat plate serrated trailing edge. The chord length $(C)$ of the aerofoil is $150 \mathrm{~mm}$, and the widths are $450 \mathrm{~mm}$ and $300 \mathrm{~mm}$ for the tests in ISVR and Brunel, respectively. From the leading edge $x / C=0$, to $x / C=0.79$ is the original NACA0012 aerofoil body, where $x$ is the streamwise direction. Further downstream, $0.79<x / C<1.0$, is a section that can be removed and the serration geometry to be attached. The serrated trailing edge used in this study is exactly the same as the previous studies in ${ }^{7,8}$ for S3, which has the following parameters as per the Fig. 1: $2 h=20 \mathrm{~mm}, \varphi=25^{\circ}, \lambda / h=1.87$ and $\varepsilon=$ $5.7 \mathrm{~mm}$. As documented in ${ }^{7}$, the tonal noise level produced by S3 is the lowest due to the low spanwise coherence in the wake, whilst a considerable broadband noise reduction across a wide range of frequency can still be achieved. Therefore the S3 serrated trailing edge is suitable for the current proof of concept study. Another reason to choose the S3 serrated trailing edge is that the sawtooth's surface area is the largest, thus potentially offering a simpler way to implement the plasma actuators to the aerofoil model.

Prior testing on the NACA0012 aerofoil, a flat plate with a 2D blunt trailing edge of $6 \mathrm{~mm}$ was investigated first. The bluntness of the flat plate is similar to the blunt root of the S3 serration. The leading edge of the flat plate has that of the NACA0012 aerofoil, whilst the width and length are the same. A steel plate is embedded to strengthen the flat plate model. The schematic of the flat plate model is shown in Fig. 2. 
Due to the restriction of the ionic wind speed generated by the plasma actuators, the investigation was only conducted at a free jet velocity of $7.5 \mathrm{~ms}^{-1}$. Rough sandpaper is attached near the leading edge at $x C \approx 0.2$ on both surfaces of the flat plate and the aerofoil to trip the boundary layers into turbulent artificially. The angle of attack for the aerofoil is $0^{\circ}$ for all cases.
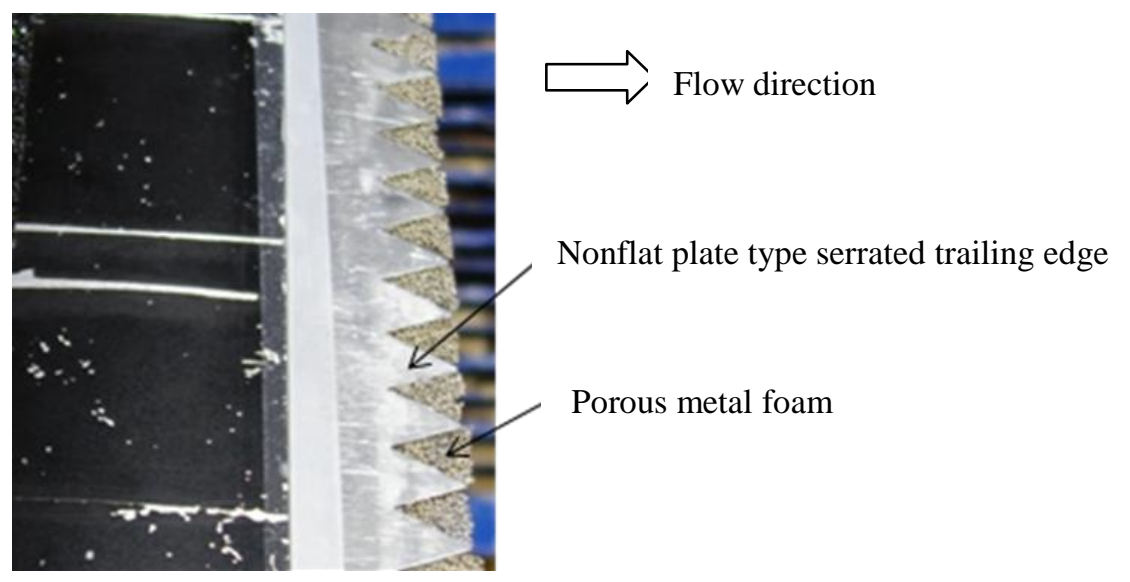

Figure 3. The new aerofoil concept with the porous metal foams attach to the interstice between adjacent members of the trailing edge serration.

\section{Porous metal foam}

As shown in Fig. 3, the interstices between adjacent members of the sawtooth across the aerofoil span were filled with metal foam porous barriers. These metal foams were precision cut to match the contour of the aerofoil so that the overall geometry of the aerofoil at the trailing edge is retained. The foamed metal is supplied by Recemat. The solidity ratio is about $5 \%$.

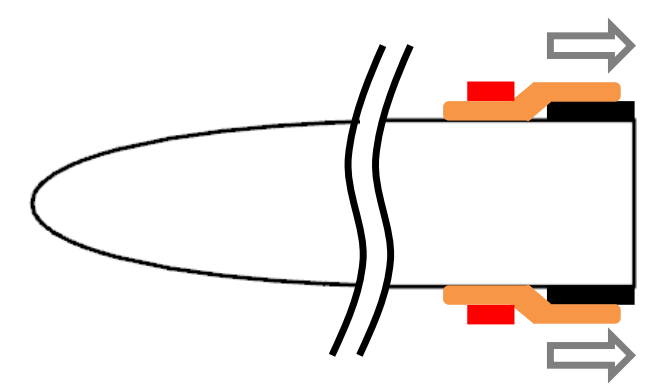

(a) FP-PA1
Exposed electrode

Dielectric layers

- Grounded electrode

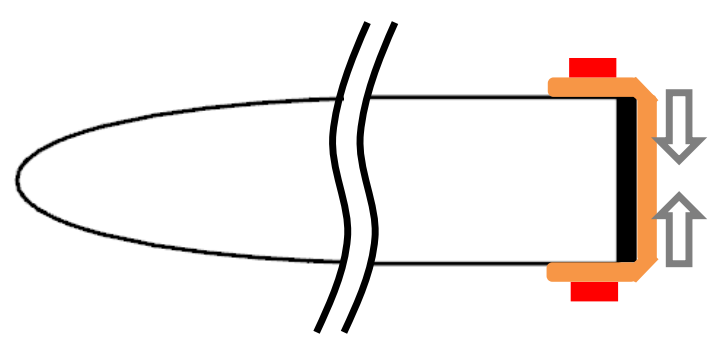

(b) FP-PA2

Figure 4. Configurations of the plasma actuators on the flat plate model

\section{Plasma actuators}

For the flat plate model, two plasma actuator configurations are adopted. The dimensions of the plasma actuators were determined with reference to the previous researches ${ }^{11-13}$. The first configuration (FP-PA1), which is shown in Fig. 4a, is expected to generate wind in the same direction as the freestream. The width of the exposed electrodes and the grounded electrodes are $5 \mathrm{~mm}$ and $10 \mathrm{~mm}$, respectively. The second configuration of the plasma actuator 
(FP-PA2) is shown in Fig. 4b. FP-PA2, which has a similar configuration as that of ${ }^{14}$, is designed to deflect the flow in an opposing direction vertically near the blunt face (see Fig. 4b). The width of the exposed electrodes for FP-PA2 is $5 \mathrm{~mm}$ but the width of the grounded electrode is $6 \mathrm{~mm}$ due to the thickness of the flat plate. All the electrodes are made of copper tape for which the thickness is about $0.035 \mathrm{~mm}(0.065 \mathrm{~mm}$ including adhesive $)$. Three and four layers of polyimide tape, each with a $0.025 \mathrm{~mm}$ thickness, are used as the dielectric material.

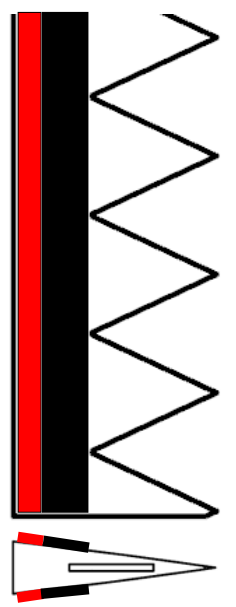

SA-PA1-1

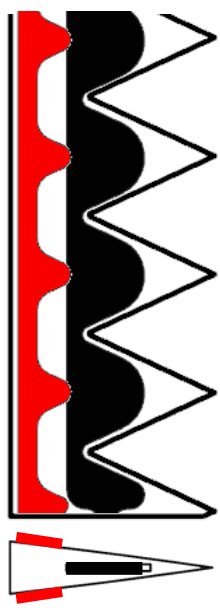

SA-PA1-2

(a) Inducing flow tangential to the airfoil surface

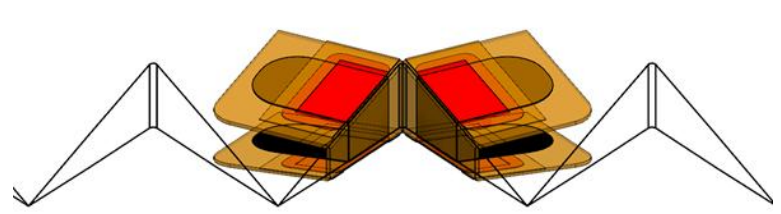

SA-PA2

(b) Inducing flow normal to the airfoil surface

Figure 5. Configurations of the plasma actuators on the aerofoil model with a nonflat plate serrated trailing edge

Similar concepts are also adopted for the aerofoil case. As sown in Fig. 5a, the FP-PA1 type was further developed into two variants for the serrated trailing edge. The first variant (SA-PA11) has the same configuration as FP-PA1 but is located at $1 \mathrm{~mm}$ upstream from the root of the serration. The second variant (SA-PA12) has the grounded electrode embedded and aligned with the sawtooth shape. The two configurations depicted in Fig. 5a are therefore a demonstration of how the tangential flow with respect to the aerofoil surface could be generated by the plasma actuators. Another configuration (SA-PA2), which is similar to the FP-PA2, is designed to generate vertical flow along the side face of the sawtooth as shown in Fig. 5b. The grounded electrodes are attached on the side surface, upper and lower surfaces of each sawtooth. The grounded electrodes are covered by four layers of dielectric over which the exposed electrodes are then attached. One layer of dielectric further wraps the edges of each exposed electrode except for the edge facing the side surface of the serration. The grounded electrodes are separated along the root of the serration to make it easier to be attached and wrapped with the dielectric layers. Due to the complex layout of the plasma actuators, the thickness of the plasma actuators is estimated to vary between $0.5 \mathrm{~mm}$ and 0.9 $\mathrm{mm}$ for the array of sawtooth.

The plasma actuators are connected to a high voltage transformer, Minipuls 6, which is enclosed in an aluminum case, whose output signals has a shape of sine wave whose amplitude can be increased up to $20 \mathrm{kV}$ peak and frequency ranges from 5 to $20 \mathrm{kHz}$. The input power is supplied by a DC power supplier $\left(\right.$ PeakTech $\left.^{\circledR} 1535\right)$ which has the maximum DC voltage of $32 \mathrm{~V}$ and the corresponding current of $20 \mathrm{~A}$. In order to vary the frequency of the high voltage output, a square wave with the same frequency generated by a function generator (LeCroy Wave Station 2012) is used. The Minipuls 6 has two monitoring ports for output voltage and current. The signals from the ports are monitored with an oscilloscope. Note that the output voltage and resonant frequency from the high voltage transformer could be affected by the capacitance of the load, which in the current experiments is the plasma actuator. Therefore, the output voltage varies with frequency as well as the configuration of the plasma actuator. For example, the resonance frequency for the FP-PA1 configuration is identified to be around $4 \mathrm{kHz}$. 
(a)

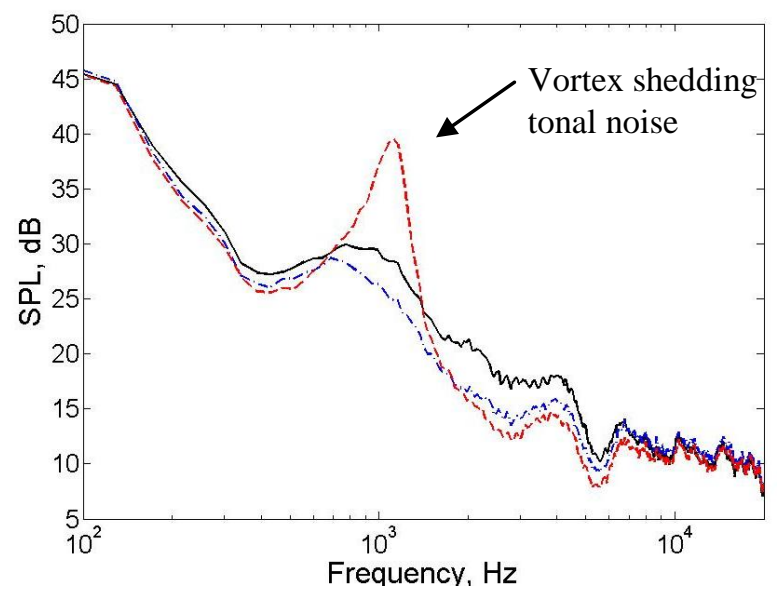

(b)

$\triangle \mathrm{SPL}$

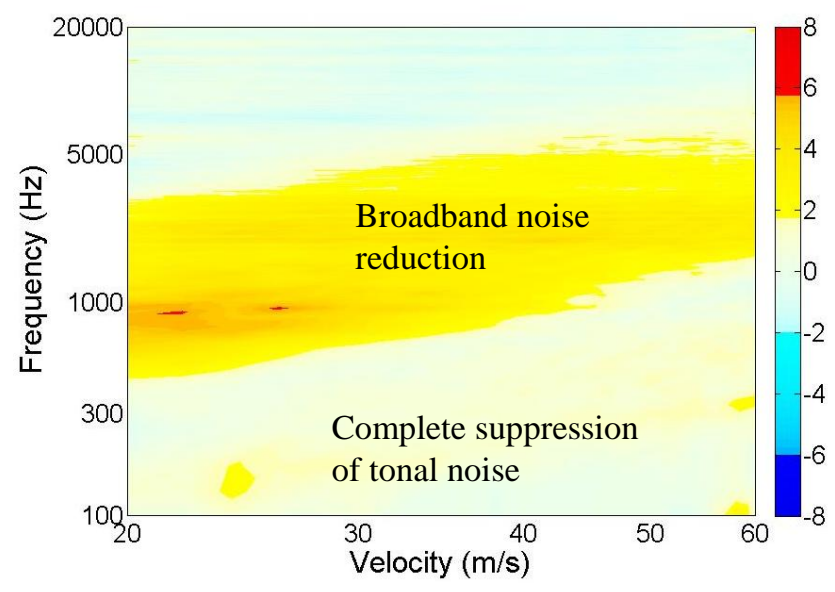

Figure 6. (a). Comparison of the Sound Pressure Level (SPL), dB, between the baseline sharp trailing edge (-), the nonflat plate type serrated trailing edge without porous metal foams (---) and with porous metal foams filling the interstices (--) of a NACA0012 aerofoil. The aerofoil is set at zero degree angle of attack and at free-jet velocity of $38 \mathrm{~ms}^{-1}$; (b). The corresponding colormap of the $\triangle \mathrm{SPL}, \mathrm{dB}$ across a wide range of flow velocity. Note that the yellow to red colours denote noise reduction; and the blue colour denotes noise increase.

\section{Results}

\section{A. Nonflat plate type serrated trailing edge with porous metal foams}

Example of the variation of Sound Pressure Level (SPL) with frequency for the aerofoil turbulent broadband noise is shown in Fig. 6a. The figure demonstrates that aerofoil trailing edge serrations cut into the main body of the aerofoil has a substantial effect on the sound spectrum. As discussed earlier, the obvious disadvantage with the use of serrations cut into the airfoil body is that it will inevitably generate narrowband noise due to vortex shedding from the root of the serration. At frequencies above the shedding frequency, broadband noise reduction can be observed. This section will demonstrate that the impact of the vortex shedding tonal noise can be significantly reduced whilst retaining the broadband noise benefits at other frequencies by the use of porous metal foams to fill the interstices between adjacent members of the sawtooth.

At a free jet velocity of $38 \mathrm{~ms}^{-1}$ in Fig. 6a, the noise spectrum produced by the new concept aerofoil is lower at frequencies between $300 \mathrm{~Hz}$ and $5000 \mathrm{~Hz}$ if compared to that produced by the aerofoil with a sharp trailing edge. When the comparison is made between the nonflat plate type serrated trailing edge with and without the porous metal foams, it is obvious that the tonal noise has been significantly reduced, indicating that the vortex shedding in the near wake is suppressed. The noise increase at high frequency, as reported in the case of flat plate type serrated trailing edge $\mathrm{e}^{1,2}$ due to the "leakage flow" between the adjacent members of the sawtooth, is not observed in the new concept aerofoil. The level of broadband noise reduction for the nonflat plate serrated trailing edge with metal foams is slightly lower than that without the metal foams (less than $1 \mathrm{~dB}$ ).

Similar results are also observed at other free jet velocity. Figure $6 \mathrm{~b}$ shows the colormap of the difference in the Sound Pressure Level ( $\Delta$ SPL) across free jet velocity between 20 and $60 \mathrm{~ms}^{-1}$. The $\Delta$ SPL is defined as the difference in Sound Pressure Level between the noise radiated by the sharp trailing edge and the nonflat plate serrated trailing edge filled by porous metal foams. Whilst the vortex shedding noise is completely suppressed by the nonflat plate serrated trailing edge with porous metal foams, significant broadband noise reduction (up to $7 \mathrm{~dB}$ ) can constantly be achieved. The improvement in the noise reduction capability for the new concept aerofoil is mainly based on the suppression of the vortex shedding in the near wake. Although not yet investigated thoroughly, the absence of the vortex shedding could be due to the convoluted and corrugated fluid pathways within the metal foam. However, it is very interesting that by filling the interstices with the porous metal foam, the seemingly lost in the serrated pattern could still produce significant broadband noise reduction. 


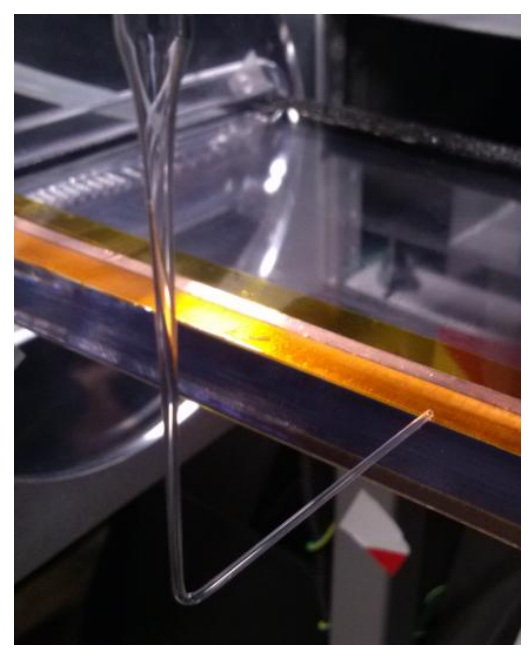

Figure 7. The glass Pitot tube for the measurement of the velocity induced by PA1 near the trailing edge of the flat plate model

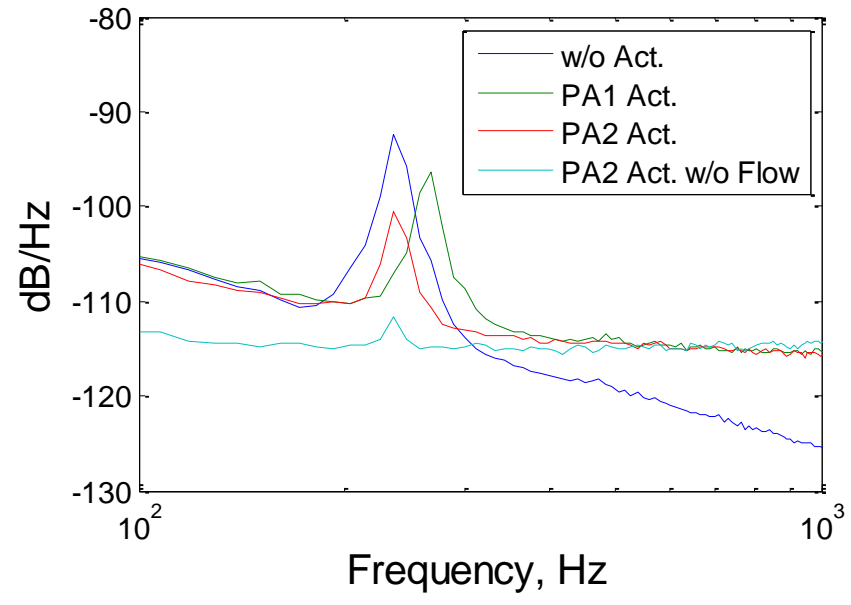

Figure 8. Comparison of the noise spectral for the baseline (no plasma is applied), FP-PA1 and FP-PA2 plasma actuators for the flat plate model.

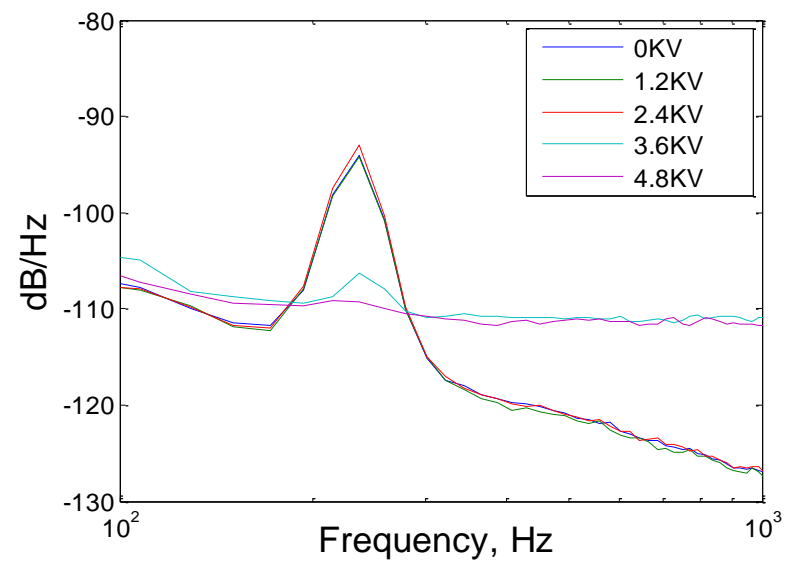

Figure 9. Comparison of the noise spectral for the baseline (no plasma is applied) and FP-PA2 plasma actuators at different output voltages for the flat plate model.

\section{B. Nonflat plate type serrated trailing edge with plasma actuators}

Velocities induced by FP-PA1 installed on the trailing edge of the flat plate without external flow were measured with a glass Pitot tube near the trailing edge (see Fig. 7). During the measurement, the input voltage was fixed at 32 $\mathrm{V}$ while the excitation frequency varies. The maximum induced velocity is found to be about $4.0 \mathrm{~ms}^{-1}$ at the excitation frequency of $8 \mathrm{kHz}$. It is know that the plasma could grow far beyond the saturation condition ${ }^{13}$. In this case, though the filamentary structures between the two electrodes continue to grow with increasing input voltage, there is no real term in the momentum gain.

For the flat plate case, noise measurements were taken at free jet velocity of $7.5 \mathrm{~ms}^{-1}$. Comparison of the noise spectral for the baseline (no plasma is applied), FP-PA1 and FP-PA2 cases are shown in Fig. 8. A tonal peak is apparent at about $236 \mathrm{~Hz}$ for the baseline case. The corresponding Strouhal number is 0.19 (or 0.20 if the thickness of the plasma actuators is taken into account). This indicates that the tonal peak is related to the vortex shedding due to the blunt trailing edge. When the FP-PA2 plasma actuators are turned on, the broad peak can be seen to decrease by about $10 \mathrm{~dB}$. The FP-PA1 plasma actuators also reduce the level of the broad peak, albeit at a lower level of 
(a)

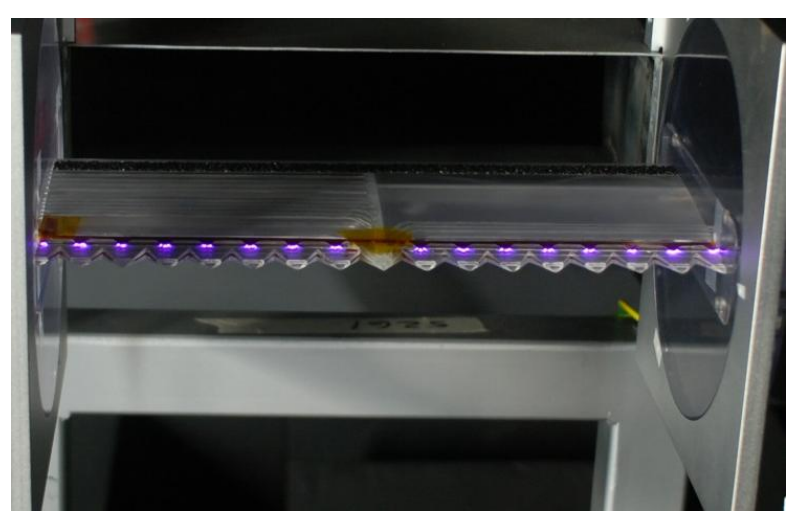

(b)
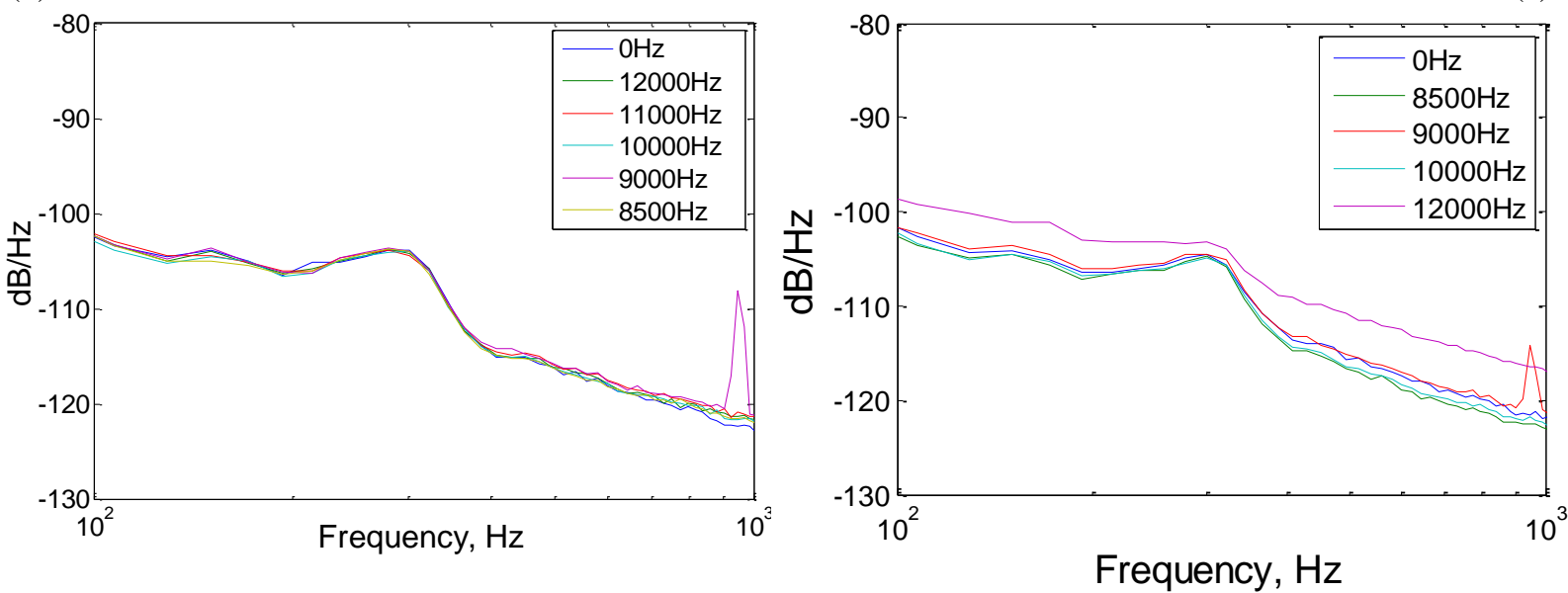

(c)

Figure 10. (a). Photo showing the plasma charges for the SA-PA1-2 configuration; (b).

Comparison of noise spectral between the baseline and SA-PA1-1 plasma configuration; and (c).

Comparison of noise spectral between the baseline and SA-PA1-2 plasma configuration.

noise reduction. It is also found that the tonal frequency has been shifted by the FP-PA1 actuators. In Nati et al. ${ }^{4}$, it was found that the FP-PA2 type of plasma actuators reduces the energy of the first and the second mode of the Karman vortex more efficiently than the FP-PA1 type of plasma actuators. We need to point out that the plasma actuators produce self noise across a large range of frequency. The effect is more significant at low free jet velocity, when the background noise is naturally low. The plasma actuators tested in the present experiment are thought to generate larger broadband noise as well as tonal noise than other study ${ }^{15}$ where only tonal peaks could be found.

Based on the results in Fig. 8, the FP-PA2 configuration seems to be more effective than FP-PA1. Using the FPPA2 configuration, it can be seen from Fig. 9 that when the output voltage (at an excitation frequency of $8 \mathrm{kHz}$ ) is below $2.4 \mathrm{kV}$, the radiated noise spectral are not much different with the baseline case. However, above $3.6 \mathrm{kV}$, the radiated noise spectral exhibit a significant reduction of the tonal noise by up to $15 \mathrm{~dB}$. Again, the reduction in the tonal noise level is also accompanied by an increase of the radiated noise at other frequencies due to the self noise of the plasma actuators.

The radiated noise spectral as a result of applying the tangential plasma actuators near the serrated trailing edge of the aerofoil case is now discussed. Figure 10a shows a photo depicting the plasma charges which is generated at around the protrusion part of the tangential actuator. Figure 10b demonstrates the radiated noise spectral of the SPPA1-1 configuration of tangential plasma actuators. The results were taken at different input frequencies that are ranged from $8.5 \mathrm{kHz}$ to $12 \mathrm{kHz}$ with the aerofoil set at zero angle of attack at a slightly higher free jet velocity of 10 $\mathrm{ms}^{-1}$. It can be seen that this configuration has no effect on the tonal noise, indicating that the vortex shedding emanated from the serration blunt roots was not suppressed. The reason behind this could be due to the limited output voltage that is dependent on the thickness of the dielectric material that covers the grounded electrode. This limitation reduces the strengths of the induced electric wind. The result of the second tangential configuration that has wavy protrusions that are aligned with the edge of the grounded electrode (SP-PA1-2) is presented in Fig. 10c. It 
(a)

(b)

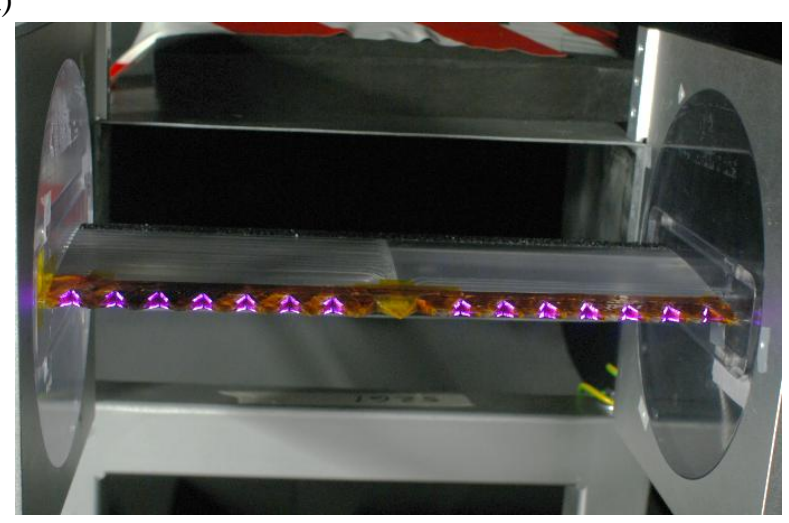

(c)
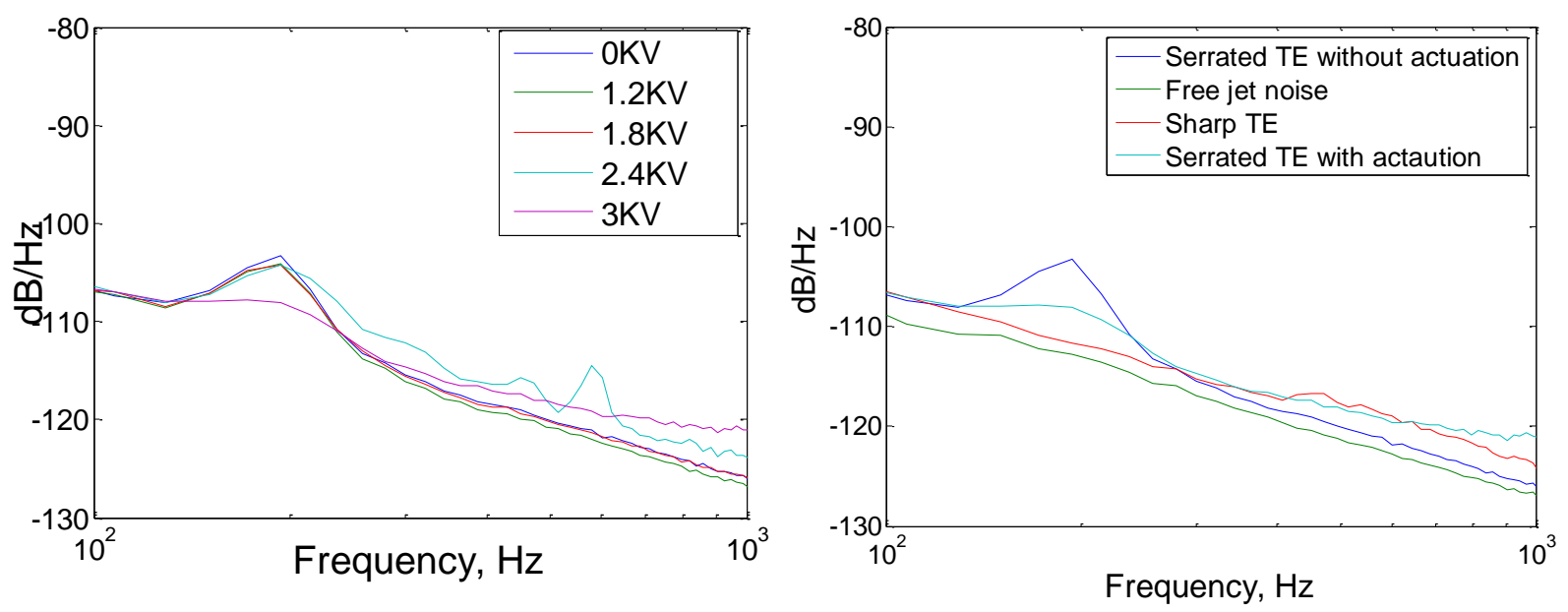

Figure 11. (a). Photo showing the plasma charges for the SA-PA2 configuration; (b).

Comparison of noise spectral between the baseline and SA-PA2 plasma configuration; and (c)

Comparison among the trailing edge noise of aerofoil with sharp, serration without plasma actuation and serration with SA-PA2 plasma actuators.

can be seen that there is an increase in the noise when the plasma actuators were operated at $12 \mathrm{kHz}$. At lower excitation frequencies, little reduction in the tonal peak can be seen. The above results demonstrate that neither the SP-PA1-1 and SP-PA1-2 configurations are effective in suppressing the vortex shedding emanated from the serrated blunt roots. This is consistent with the previous flat plate results.

The plasma charges generated at the blunted sides of each sawtooth in SA-PA2, which aims to produce downward electric winds, is shown in Fig. 11a. Figure 11b shows the corresponding radiated noise spectral of the serrated aerofoil. The free jet velocity is set at $7.5 \mathrm{~ms}^{-1}$ and the angle of attack is again at zero degree. It can be seen that the shape and position of both the exposed and grounded electrodes can play an important role in the serrated trailing edge noise reduction. A good level of tonal noise reduction of about $5 \mathrm{~dB}$ at output voltage of $3 \mathrm{kV}$ and excitation frequency of $8 \mathrm{kHz}$ can be achieved. Fig. 11c shows a comparison among the trailing edge noise of aerofoil with sharp, serration without plasma actuation and serration with SA-PA2 plasma actuators. When compare with a sharp trailing edge, the use of a nonflat plate type serrated trailing edge can produce an extra tonal component (due to the vortex shedding), but with lower broadband noise level at high frequencies (due to the serration effect). In the figure, it can be seen that in addition to the tonal noise reduction, the SA-PA2 plasma actuators can cause some deteriorations of the serration effect on the broadband noise reduction. 


\section{Conclusion and outlook}

This paper presents the results on the use of porous metal foams (passive control) and dielectric barrier surface plasma actuations (active control) for the reduction of vortex shedding tonal noises from the nonflat plate type trailing edge serration in a NACA0012 airfoil.

The use of porous metal foams to fill the interstices between adjacent members of the sawtooth can almost completely suppress the vortex shedding tonal noise, whilst the serration effect on the broadband noise reduction is retained. This concept will promote the nonflat plate type serrated trailing edge to become a genuine alternative to the conventional flat plate type serrated trailing edge, which is known to have drawbacks in the structural stability, aerodynamic performances and implementation issues.

For the plasma actuators, there are still many rooms for improvement. The generic "PA1" configuration, which produces electric wind in a tangential direction, is not very effective in suppressing the vortices emanated from the serration blunt root. On the other hand, for the "PA2" configuration which produces electric wind in a vertical direction, good level of vortex shedding tonal noise reduction has been demonstrated. However, the self noise produced by the plasma actuators negates the noise benefits on the tonal noise reduction. This characteristic illustrates the need to further develop the plasma actuators in a two pronged approach:

1. Increase the electric wind speed, thereby allowing the plasma actuators to be used in a higher free jet velocity which naturally produces a larger level of jet noise

2. Reduce the self noise radiated by the plasma actuators

\section{Acknowledgment}

The research is sponsored by the EPSRC grant EP/K002309/1 in the United Kingdom.

\section{References}

${ }^{1}$ Gruber, M., Joseph, P. F. and Chong, T. P., "On the mechanism of serrated airfoil trailing edge noise reduction," AIAA Paper 2011-2781, 2011, 17 ${ }^{\text {th }}$ AIAA/CEAS aeroacoustic conference and exhibit, Portland, Oregon.

2 Oerlemnans, S., Fisher, M., Maeder, T. and Korler, K., "Reduction of wind turbine noise using optimized airfoils and trailing edge serrations," AIAA Journal, Vol. 47, 2009, pp.1470-1481.

${ }^{3}$ Moreau, D. J., Brooks, L. A. and Doolan C. J., "Flat plate self-noise reduction at low-to-moderate Reynolds number with trailing edge serrations," 2011, In Proceedings of Acoustics, Gold Coast, Australia.

${ }^{4}$ Geyer, T., Sarradj, E. and Fritzsche, C., "Measurement on the noise generation at the trailing edge of porous airfoils," Experiment in Fluids, Vol. 48, 2010, pp.291-308.

${ }^{5}$ Herr, M., "Design Criteria for Low-Noise Trailing-Edges," $13^{\text {th }}$ AIAA/CEAS Aeroacoustic Conference and Exhibit, AIAA Paper 2007-3470, 2007, Rome, Italy.

${ }^{6}$ Finez, A., Jondeau, E., Roger, M. and Jacob, M. C., "Broadband noise reduction with trailing edge brushes," AIAA Paper 2010-3980, 2010, $16^{\text {th }}$ AIAA/CEAS aeroacoustic conference and exhibit, Stockholm, Sweden.

${ }^{7}$ Chong, T. P., Vathylakis, A., Joseph, P. and Gruber, M. 2013, "Self-noise produced by an airfoil with nonflat plate trailingedge serrations", AIAA J., Vol. 51, 2013, pp. 2665-2677.

${ }^{8}$ Chong, T. P., Joseph, P. and Gruber, M. 2013, "Airfoil self noise reduction by non-flat plate type trailing edge serrations", Applied Acoustics, Vol. 74, 2013, pp. 607-613.

${ }^{9}$ Chong, T. P., Joseph, P. F. and Davies, P. O. A. L., "Design and Performance of an Open Jet Wind Tunnel for AeroAcoustic Measurement," Applied Acoustics, Vol. 70, 2009, pp. 605-614.

${ }^{10}$ Vathylakis, A., Kim, J. H. and Chong, T. P., "Design of a low-noise aeroacoustic wind tunnel facility at Brunel University," $20^{\text {th }}$ AIAA/CEAS Aeroacoustic Conference and Exhibit, 2014, Atlanta, USA.

${ }^{11}$ Moreau, E., "Airflow Control by Non-Thermal Plasma Actuators," Journal of Physics D: Applied Physics, Vol. 40, 2007, pp. 605-636.

${ }^{12}$ Forte, M., Jolibois, J., Pons, J. Moreau, E., Touchard, G., and Cazalens, M., "Optimization of a Dielectric Barrier Discharge Actuator by Stationary and Non-Stationary Measurements of the Induced Flow Velocity: Application To Airflow Control," Experiment in Fluids, Vol. 43, 2007, pp. 917-928.

${ }^{13}$ Thomas, F. O., Corke, T. C., Iqbal, M., Kozlov, A., and Schatzman, D., "Optimization of Dielectric Barrier Discharge Plasma Actuators for Active Aerodynamic Flow Control," AIAA Journal, Vol. 47, 2009, pp. 2169-2178.

${ }^{14}$ Nati, G., Kotsonis, M., Ghaemi, S. and Scarano, F., "Control of vortex shedding from a blunt trailing edge using plasma actuators", Experimental Thermal and Fluid Science, Vol. 46, 2013, pp. 199-210.

${ }^{15}$ Kozlov, A., and Thomas, F. O., "Active Noise Control of Bluff-Body Flows Using Dielectric Barrier Discharge Plasma Actuators," 15th AIAA/CEAS Aeroacoustics Conference, AIAA Paper 2009-3245, May 2009. 